\title{
Blind Image Quality Evaluation of Stitched Image using Novel Hybrid Warping Technique
}

\author{
Sanjay T. Gandhe ${ }^{1}$, Omkar S. Vaidya ${ }^{2}$ \\ Department of Electronics \& Telecommunication Engg. \\ Sandip Institute of Technology \& Research Centre Nashik, India \\ Savitribai Phule Pune University, Pune, India
}

\begin{abstract}
Image stitching is collection of sequential images captured at fixed camera center having considerable amount of overlap and produces aesthetically pleasing seamless panoramic view. But, practically it is very difficult to obtain clean and pristine stitched panoramic image of particular scene as such images are apparently distorted. In this paper, a novel Hybrid Warping technique is used that combine two global warps and one local warp and helps to refine image alignment stage. Our proposed method optimizes Homography Screening to rectify problem of perspective distortion and Edge Strength Similarity approach to quantify structural irregularities. The Blind Image Quality Evaluation models such as Blind Image Quality Index (BIQI), Blind/Reference-less Image Spatial QUality Evaluator (BRISQUE) and BLind Image Integrity Notator using DCT Statistics (BLIINDS-II) are employed to measure objective quality of stitched image. The experimental results showed that blind image quality score of proposed method is significantly better than latest existing methods.
\end{abstract}

Keywords-Blind image quality evaluation; hybrid warping; image stitching; panoramic image

\section{INTRODUCTION}

Now-a-days image stitching technology is available readily in advanced digital cameras and smart phone devices. Also with launch of several commercial image stitching software, user can obtain panoramic view which has great demand in photogrammetry, medical surgery, underwater survey, UAV imagery etc. But, results obtained in above cases face one of the issues like structural misalignments, colour differences, motion blur and noticeable seams. To overcome above issues, most of the researchers have used geometric transformation, image warping algorithms and image compositing techniques.

The proposed work is focused on accurate alignment of two overlapping images using novel Hybrid Warping technique. On other side, many warping models such as APAP [1], SPHP [2], ELA [3], AANAP [4] etc. produces stitched image with distinctive objects. To access the stitched image quality, standard reference image plays an important role. If pristine reference image is available and quality of distorted image is compared against it, such type of model is known as full reference image quality (FR IQA) model. In case of image stitching application, the resulting image of above mentioned warping models are apparently distorted and hence standard reference images are unavailable. Therefore, blind image quality evaluation (i.e. no-reference image quality model) is introduced which does not require reference image to measure objective quality of stitched image. The score obtained from

This work is financially supported by Savitribai Phule Pune University, India under Research Mentorship Program Scheme (18TEC001416). blind image quality models address the issues like severity of perceptual distortion, loss of visual information due to blurring and loss of resolution due to limited depth of field.

In this paper, the growth of successive image stitching algorithm is surveyed in Section II. Section III described novel hybrid image warping technique for image stitching in detail. Section IV covered different blind image quality models with mathematical representations. The parameter setting of proposed method and comparative analysis with other image stitching method is given in Section V. At last, conclusion is drawn in Section VI.

\section{REVIEW OF RELATED WORK}

In order to obtain seamless panoramic image stitching result, considerable efforts have been taken by many researchers in last few decades which are reviewed below:

Richard Szeliski described common pipeline of image stitching which are image alignment techniques, 2-D motion models, 3-D transformations and image blending algorithms in [5]. Brown reviewed important distinction, feature spaces, similarity metrics and search strategies used in image registration [6]. Chen laid down fundamental idea behind image stitching in [7] and evaluated combinations of image registration and merging methods. The detection and description of keypoint features from images is key step in image stitching. Authors in [8]-[14] proposed several feature extraction techniques and demonstrated comparative analysis by keeping in view of proper image registration. Fischler and Bolles introduced statistically robust fitting model named RANSAC in [15] which finds correct feature matches (i.e. inliers) between two images with overlap. However in case of multi-structure data, probability of hitting all inliers is main challenge. To achieve this issue, authors in [16] used multiguided sampling algorithm to accelerate hypothesis generation and require less CPU time as compared to RANSAC.

To align two images of planar scene and preserve projective nature of registered image, 8 parameters $3 \times 3$ homography matrix is useful. But, if images are acquired from different viewpoint and transformed other than rotation, then alone homography transformation is not sufficient to align the images [6], [17].

Authors in [18] used two homographies; one for distant plane (i.e. objects far away from camera) and another for ground plane (i.e. objects closer to camera) to register pair of images. If in case of arbitrary scenes (i.e. background and 
foreground plane is unable to separate), then stitching is unsuccessful. Wen-Yan Lin et al. used 6 parameters affine transformation matrix. In [19], smoothness is employed to extrapolate non-overlapping region and produced protrusion free stitched image. But, due to limited degree of freedom distortions occurred in non-overlapping regions of stitched image.

In [1], the source image is divided into mesh which consists of group of pixels. From each mesh, central positioned coordinate is warped using global homography. This produces projective warp that fits in overlapping region to obtain accurate alignment. This approach is called as Moving Direct Linear Transform (MDLT). This method accurately aligns objects in overlapping region but produces excessive enlargement in non-overlapping region in source image.

To resolve above issue, authors constructed shape preserving projective warps (SPHP) in [2]. Here, from pair of images global homography is estimated and then $\mathrm{u}-\mathrm{v}$ coordinates are derived. The projective warp is divided into two half spaces; on one half space similarity transformation used for extrapolation and on other half space projective transformation is performed for alignment purpose. Still, problem of parallax is unresolved with this. This method is more meaningful if combined with APAP method.

To alleviate the problem of parallax, researcher proposed elastic local alignment (ELA) model and Bayesian feature refinement model in [4]. This method has better computational complexities as compared with above mentioned methods. In case of severe occlusion, this method gives undesired results.

As per as objective quality of stitched image is concerned, very few research attention is paid to blind image quality assessment of panoramic view. Recently, Suiyi Ling et al. proposed no reference convolutional sparse coding using trained kernel capture distortion in local region of stitched image [20].

\section{NOVEL Hybrid IMAGE WARPING TECHNIQUE}

The mathematical representation of proposed Hybrid Warping model is submitted in our previous work [21]. In overlapping area of two images, the moving direct linear transform warp is employed while differentiating local Homography (i.e. smoothened) is achieved by computing weights in non-overlapping region. Finally global similarity transformation is applied to entire image to have regular perspective panoramic scene. The dataflow of proposed work is shown in Fig. 1.

The main contribution of our research work is as follows:

The term gamma $(\gamma)$ is offset parameter and its value is in range 0 and 0.1 . If the value of $\gamma=0$, then stitched image has wavy effects because of its fixed nature in Gaussian Weighting. Also, if value is kept 1, then warp loses its flexibility. Similarly, the scale parameter $(\sigma)$ value depends on image size to get better stitched results. Therefore, the value of $\gamma$ is optimized in range of 0 to 1 and that of and $\sigma$ is from 8.5 12. To minimize perspective distortion, calculation of average weights $[a, b, c, d]$ is carried out with respect to previous grid value and assigned it to similarity transformation and local moving DLT warp.

Another approach is Homography Screening in which homographies are filtered depending on its sum of distances between four corner values (i.e. $C_{i}$ and $\overline{C_{i}}$ ). From image initially four corner points $\mathrm{C}_{\mathrm{i}}$ are obtained and by its respective homography its corresponding four corner points $\overline{\mathrm{C}_{i}}$ are calculated. According to eq. (1) best fitting similarity transformation $\left(\mathrm{H}_{\mathrm{s}}\right)$ is computed.

$$
\mathrm{H}_{\mathrm{s}}=\arg \min _{\mathrm{H}_{\mathrm{s}}} \sum_{\mathrm{C}_{\mathrm{i}}}\left\|\mathrm{H}_{\mathrm{s}} \mathrm{C}_{\mathrm{i}}-\overline{\mathrm{C}}_{\mathrm{i}}\right\|^{2}
$$

The sum of distances between $\mathrm{C}_{\mathrm{i}}$ and $\overline{\mathrm{C}}_{\mathrm{i}}$ are normalized and if this value is more than specified threshold value (i.e. 0.001), then such homographies are discarded.

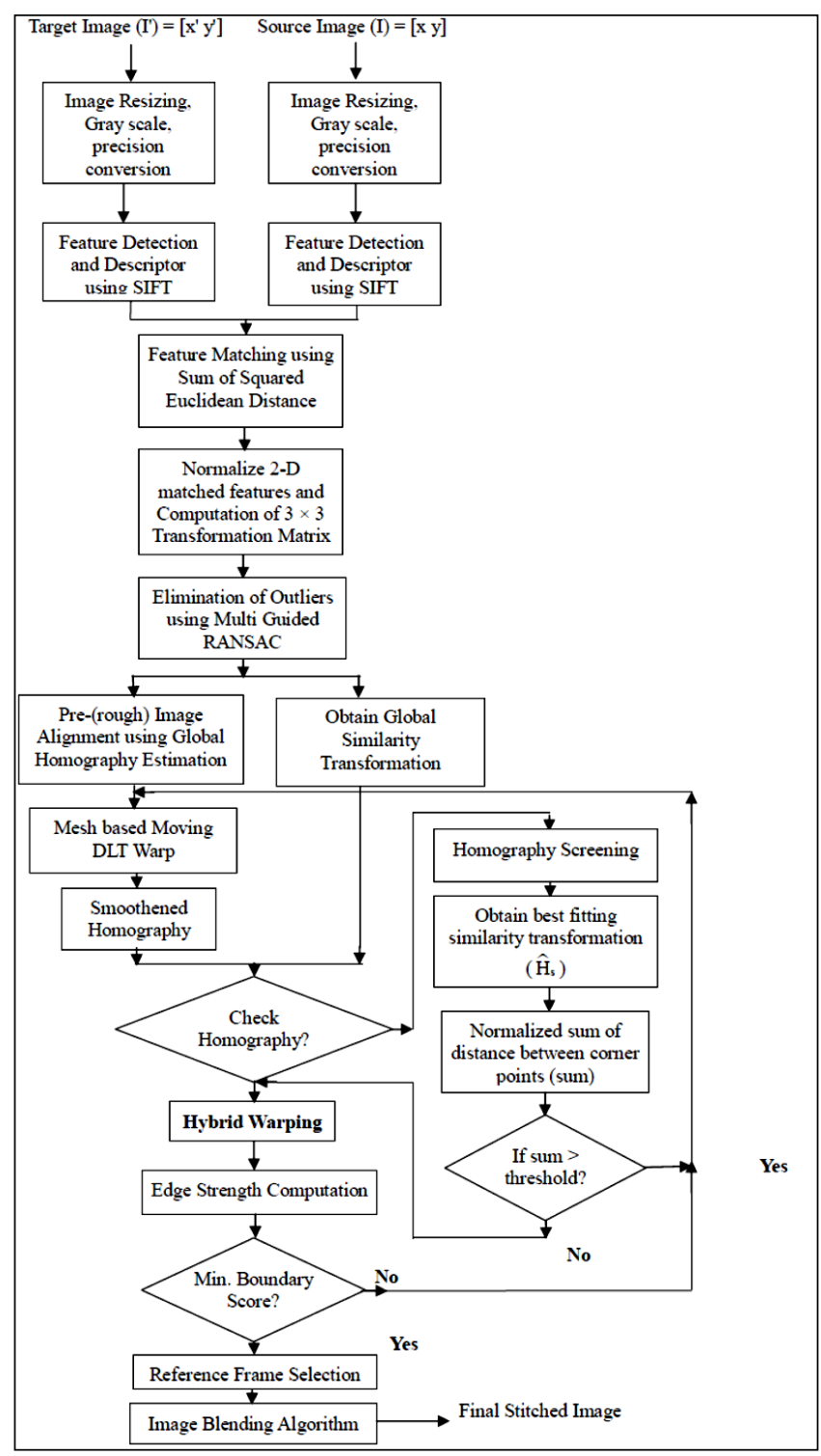

Fig. 1. Data Flow of Proposed Method. 
One more approach is investigated in this paper based on edge structural similarity (ESSIM) to determine the boundaries in stitched image. From this, total boundary score is calculated. The minimum score value indicates less misalignment error in stitched image.

Our proposed technique is very efficient in terms of geometric realism as it passes through three improving stages viz. Hybrid Warping, Homography Screening and canny edge detection (for estimating least boundary score). To obtain planar perspective scene, rectilinear reference frame is chosen. Then to conceal colour inconsistencies and visible seams, image blending technique is employed over final warped image. Finally, image quality assessment is carried out using blind image quality evaluation models on stitched image.

\section{Blind IMAGe Quality Models}

There are mainly three approaches of Blind Image Quality models viz. (a) Distortion Specific, (b) Feature Extraction with Learning, (c) Natural scene statistics (NSS). The distortion specific approach determines the amount of distortion in image and estimates the score accordingly. In second approach, features are extracted from image and sort distorted image with undistorted one using trained learning mechanism. The NSS model determines statistical behaviour of natural image and quantifies the introduction of distortion based on distance measure or direction calculation. The use of above approaches is discussed in some of well-known blind image quality models as follows.

\section{A. Blind Image Quality Index (BIQI)}

Moorthy and Bovik proposed a two stage framework named BIQI in [22]. In first stage, image features are computed using wavelet transform. After that the presence of distortion (such as blur, additive white Gaussian noise, JPEG, JPEG2K and fast fading) is judged and classified using support vector machine. The probability of each distortion $\left(\mathrm{P}_{\mathrm{i}}\right)$ is calculated using multi class radial basis function. In second stage, to obtain image quality of image $\left(\mathrm{q}_{\mathrm{i}}\right)$ towards these distortions, support vector regression is used. Finally, the BIQI score is given in eq. (2):

$$
B I Q I=\sum_{i=1}^{5} P_{i} \cdot q_{i}
$$

Where, $\mathrm{P}_{\mathrm{i}}=\{\mathrm{i}=1, \ldots, 5\}$ and $\mathrm{q}_{\mathrm{i}}=\{\mathrm{i}=1, \ldots, 5\}$

The BIQI score is in range of 0 to 100 , where lower value indicates best quality and higher value indicates poor quality.

\section{B. Blind/Refereneceless Image Spatial QUality Evaluator (BRISQUE)}

Furthermore, Mittal et al. developed spatial domain, opinion aware (OA), distortion aware (DA) NSS based model named BRISQUE in [23]. It is transform free (i.e. do not require mapping of data from one form to other especially DCT or wavelet). It is. The development of BRISQUE model is given below:

Consider Image $\mathrm{I}(\mathrm{m}, \mathrm{n})$, where $\mathrm{m}$ and $\mathrm{n}$ are spatial coordinate indicate image height and width respectively. The local non-linear operation is applied to image is given in eq. (3)

$$
\hat{I}(m, n)=\frac{I(m, n)-\mu(m, n)}{\sigma(m, n)+C}
$$

Where, $\mu(i, j)$ is local mean and $\sigma(i, j)$ is local variance (highlight object boundaries and high contrast).

$$
\begin{aligned}
& \mu(m, n)=\sum_{k=-K}^{K} \sum_{l=-L}^{L} \omega_{k, l} I_{k, l}(m, n) \\
& \sigma(m, n)=\sqrt{\sum_{k=-K}^{K} \sum_{l=-L}^{L} \omega_{k, l}\left(I_{k, l}(m, n)-\mu(m, n)^{2}\right.} \\
& \quad \omega=\left\{\omega_{k, l} \mid k=-3, \ldots 3, l=-3, \ldots 3\right\} \quad \text { is Gaussian weighting }
\end{aligned}
$$

function. $\mathrm{K}$ and $\mathrm{L}$ are standard deviations.

The presence of distortion is judged by finding the mean subtracted contrast normalized (MSCN) coefficients. To observe the changes in coefficient, generalized Gaussian distribution (GGD) with zero mean is used. It is given in eq. (6).

$$
f\left(x ; \alpha, \sigma^{2}\right)=\frac{\alpha}{2 \beta \Gamma(1 / \alpha)} \exp \left(-\left(\frac{|x|}{\beta}\right)^{\alpha}\right)
$$

Where, $\beta=\sigma \sqrt{\frac{\Gamma(1 / \alpha)}{\Gamma(3 / \alpha)}}$ is scale parameter and $\Gamma(\cdot)$ is gamma function.

From GGD, the shape factor $(\alpha)$ and variance $(\sigma 2)$ are computed to obtain feature set and such feature sets are helpful to quantify the distortion in image. The distribution of MSCN coefficient is homogeneous in case of clean distortion- free image and thus neighborhood of MSCN coefficient is modeled along four dimensions like vertical, horizontal, two diagonals. At last all extracted features are mapped into quality score with the help of support vector machine regressor (SVR). The quality score is computed in range of 0 and 100; lower value indicates good quality and higher value is poor in terms of image quality.

\section{BLind Image Integrity Notator using DCT Statistics (BLIINDS-II)}

Saad and Bovik introduced a model which extracts features like contrast, structural information, sharpness and orientation anisotropies with the help of discrete cosine transform [24]. To obtain image contrast, 2-D DCT is applied to local patch of 17 $\times 17$ size. The final value is averaged from all patches prior to normalization. From the non-DC DCT frequency coefficient, the structural information is retrieved. It is observed the peak values especially at zero position (i.e. magnitude of coefficient on X-axis) on histogram in case of distorted images. To find the degree of peak values of histogram, kurtosis is used which is given in eq. (7).

$$
\kappa(x)=\frac{E(x-\mu)^{4}}{\sigma^{4}}
$$

Where, $\mu$ is mean of $\chi$ while $\sigma$ is standard deviation. 
The value of kurtosis is considered as lowest 10th percentile of obtained values. To evaluate scene's directional information due to degradation process, then it is necessary to calculate orientation anisotropy. Such features are measured using Renyi entropy. Firstly, 1-D DCT patch is considered and along four orientations (i.e. $\theta=0^{\circ}, 45^{\circ}, 90^{\circ}, 135^{\circ}$ ) DCT image patches are computed. The normalized DCT coefficient with local patch is given in eq. (8).

$P_{\theta}[n, k]=\frac{P_{\theta}[n, k]^{2}}{\sum_{k} P_{\theta}[n, k]^{2}}$

Where, $\mathrm{n}$ is spatial index and $\mathrm{k}$ is frequency DCT coefficient. The Renyi entropy of eq. (8) is given in eq. (9).

$R_{\theta}[n]=-\frac{1}{2} \log \left(\sum_{k} P_{\theta}[n, k]^{3}\right)$

Collectively for every image patch average per orientation is given by $E[R \theta]$. The value of anisotropy is maximum of variance across four orientation is given by $\operatorname{var}(\mathrm{E}[\mathrm{R} \theta])$.

After calculating all features, the quality score is determined using prediction model viz. multivariate Gaussian and multivariate Laplacian distribution. The BLIINDS-II score is in range 0 to 100 and lower value indicates good quality and higher value means degraded quality.

\section{EXPERIMENTATION}

To validate the effectiveness of proposed method, the experiment is implemented on Intel Processor (Corei3) having $4 \mathrm{~GB}$ physical memory and $2 \mathrm{GHz}$ CPU speed. The evaluation is performed in MATLAB 2017a combined with Computer Vision toolbox. To assess the blind image quality metrics, the standard image dataset is used especially for image stitching application and compared our results with As Projective As Possible (APAP), Adaptive As Natural As Possible (AANAP), Shape Preserving Half Projective (SPHP) and Elastic Local Alignment (ELA) methods.

There are different image sizes used from $240 \times 320$ to 898 $\times 1197$ pixels. From pair of input image, initially features are extracted using Scale Invariant Feature Transform (SIFT) and matched such keypoints using SIFT descriptor. The computations of dense features are possible with the help of open source portable library named VLFeat [25] which is suitable for speed optimization. After extraction, keypoint matching between two images is done based on squared Euclidean distance. The default threshold is set to 1.5. To obtain fundamental matrix and estimate homography matched position keypoints are normalized. The multi-guided RANdom SAmple Consensus (RANSAC) fits the maximum number of inliers by comparing threshold value of 0.1 . Further in mesh based MDLT warping, the grid size is kept as $100 \times 100$ cells.

For fair comparison, blind image quality models are employed on output stitched images of APAP, AANAP, SPHP, ELA and our proposed method.

\section{A. Quantitative Comparison}

The quality score obtained by blind image quality models such as BIQI, BRISQUE and BLIINDS-II is depicted in Table I.

TABLE I. COMPARISON AMONG STATE-OF-THE-ART STITCHING METHODS BASED ON BLIND IMAGE QUALITY EVALUATION SCORE

\begin{tabular}{|c|c|c|c|c|c|c|}
\hline \multirow{2}{*}{$\begin{array}{l}\text { Image } \\
\text { Datasets }\end{array}$} & \multirow{2}{*}{$\begin{array}{l}\text { Blind Image } \\
\text { Quality } \\
\text { Parameters }\end{array}$} & \multicolumn{5}{|c|}{ Image Stitching Methods } \\
\hline & & APAP & SPHP & $E L A$ & AANAP & Ours \\
\hline \multirow{3}{*}{ Temple } & BIQI & 21.45 & 31.47 & 39.65 & 22.82 & 10.64 \\
\hline & BRISQUE & 46.13 & 48.59 & 60 & 53.59 & 41.83 \\
\hline & BLIINDS-II & 18 & 8.5 & 21 & 16.5 & 7 \\
\hline \multirow{3}{*}{ Railtracks } & BIQI & 28.98 & 33 & 27.58 & 24.59 & 12.98 \\
\hline & BRISQUE & 43.81 & 48.59 & 63.59 & 53.49 & 40.19 \\
\hline & BLIINDS-II & 18 & 25.5 & 11.5 & 17 & 8.5 \\
\hline \multirow{3}{*}{ Rooftops } & BIQI & 26.47 & 22.84 & 2.12 & 27.57 & 15.11 \\
\hline & BRISQUE & 62.7 & 48.59 & 33 & 30.13 & 31.61 \\
\hline & BLIINDS-II & 20.5 & 40.5 & 15.5 & 13.5 & 0.7 \\
\hline \multirow{3}{*}{ Garden } & BIQI & 21.53 & 31.82 & 31.88 & 16.14 & 7.52 \\
\hline & BRISQUE & 13.18 & 48.59 & 56 & 13.18 & 35.49 \\
\hline & BLIINDS-II & 15 & 18 & 10 & 14.5 & 1.5 \\
\hline \multirow{3}{*}{ Apartment } & BIQI & 18.47 & 29.36 & 47.37 & 17.62 & 8.7 \\
\hline & BRISQUE & 20.05 & 49 & 63.59 & 35.63 & 18.35 \\
\hline & BLIINDS-II & 12 & 20.5 & 6.5 & 4 & 0.5 \\
\hline \multirow{3}{*}{ Chess-Girl } & BIQI & 24.37 & 33.28 & 36.35 & 23.8 & 11.54 \\
\hline & BRISQUE & 20.38 & 48 & 57 & 47.31 & 7.82 \\
\hline & BLIINDS-II & 12 & 8.5 & 9.5 & 7.5 & 0.75 \\
\hline \multirow{3}{*}{ Coach } & BIQI & 31.43 & 48.62 & 41.41 & 22.52 & 13.6 \\
\hline & BRISQUE & 22.59 & 44 & 62 & 33.21 & 18.55 \\
\hline & BLIINDS-II & 9 & 24.5 & 7.5 & 3 & 0.55 \\
\hline
\end{tabular}

Table I illustrate that the blind image quality assessment parameters in our proposed method give excellent result as compared with other methods for all datasets. The lower values of parameters indicate reduced content of distortion in stitched panoramic image.

The lower quality score of BIQI infers that image has lower Gaussian blur as well as less JPEG2K and JPEG compression level. For temple dataset, in case of APAP, ELA and SPHP method stitched image shows significant blur. The lower value of BRISQUE gives close relation between statistical features of stitched image to natural scene statistics. In our proposed system, BRISQUE score is lowest (except Garden and Rooftops) among all stitching methods and thus such results are highly correlated with human perception. This is due to noticeable remaining black (or white) pixels as compared with total number of pixels in stitched image of Garden and Rooftops dataset. The BLIINDS-II score exhibit most of behavior of human visual systems i.e. perceptual distortion, 
visually sensitive structural anomalies and edge impairments (especially around aligned part of stitched image). The value of BLIINDS-II score is obtained in all dataset is lowest in our proposed method, this itself speak about quality of stitched image.

\section{B. Qualitative Comparison}

Consider the target and source image of temple dataset as shown in Fig. 2(a) and (b) respectively.

Image stitching using APAP method is shown in Fig. 3. The part in red colour rectangle box showed structural misalignment and green colour rectangle box shows slant shape of building structures.

Fig. 4 shows stitched output using AANAP method. The image is highly blurred in overlapping region due to homography linearization. Also, parallax is observed in overlapping region and is highlighted by red colour rectangle box.

The stitched image using SPHP method is shown in Fig. 5. After stitching, source image is rotated and perspective nature is lost. The rectangle red box indicates severe parallax error (roof of temple) and local misalignments (ground plane).

The image stitching obtained by ELA method is shown in Fig. 6. The common structural mistakes like curved alignment of paver block and roof of temple are corrected in this method. However, the non-overlapping part is highly extrapolated like APAP. The ghosting effect is observed around the person standing on road. The temple object and buildings are not parallel with each other.

Fig. 7 shows image stitching using our proposed method. The yellow colour rectangle boxes show that accurate alignment without any parallax error and ghosting artifact. The motion blur is eliminated in overlapping part. The stitched output images shown in Fig. 3 to Fig. 7 are best viewed on computer screen.

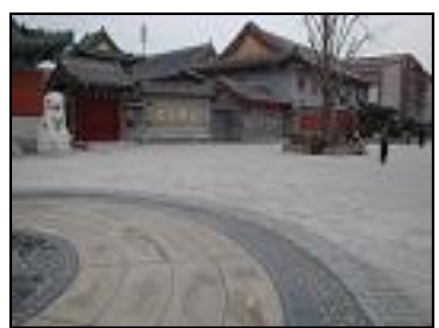

(a)

Fig. 2. (a) Target Image, (b) Source Image.

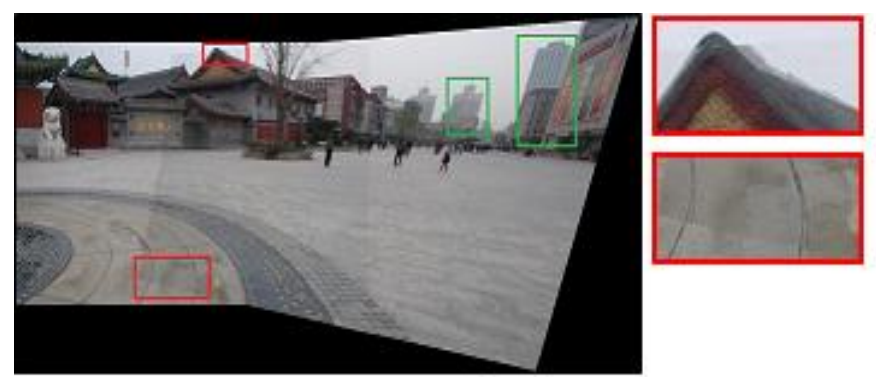

Fig. 3. Image Stitching using APAP Method.
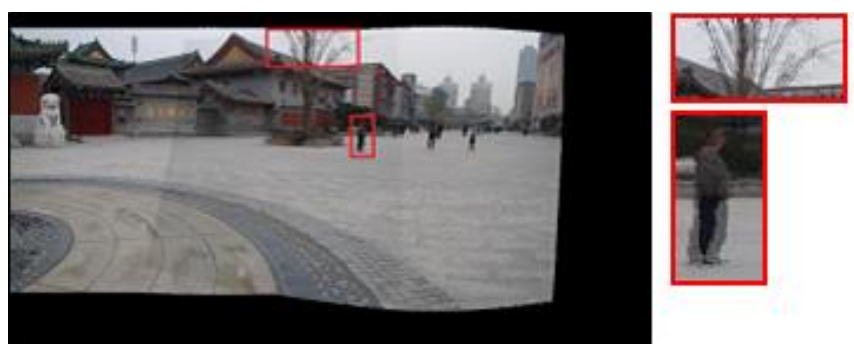

Fig. 4. Image Stitching using AANAP Method.
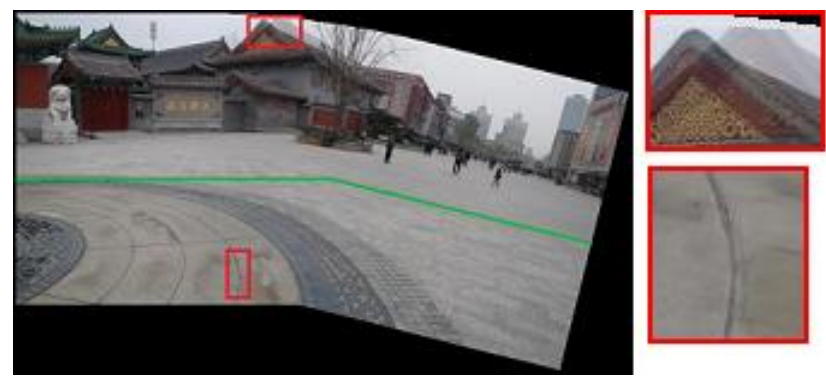

Fig. 5. Image Stitching using SPHP Method.
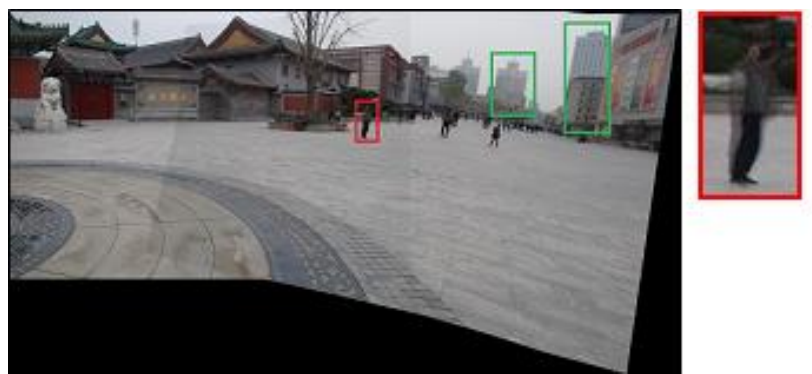

Fig. 6. Image Stitching using ELA Method.

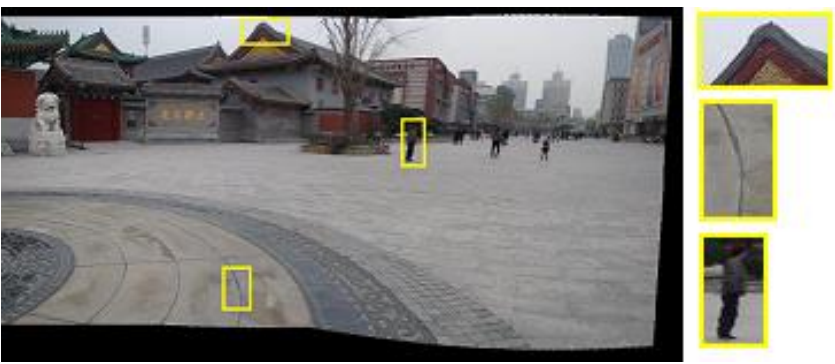

Fig. 7. Image Stitching using our Proposed Method.

\section{CONCLUSION}

A novel hybrid image stitching approach is presented in this paper. In order to obtain natural distortion-free panoramic view, an idea of homography screening and edge strength similarity measure is executed while warping the images. Later, the concept and mathematical representations of blind image quality models is explained. The quantitative analysis evidenced that our proposed method performed well among state-of-the-art stitching methods. From qualitative analysis, it is verified that stitched result produced using proposed method is free from parallax, motion blur, structural misalignment and colour inconsistencies. In future, the full reference image quality assessment will be investigated only in overlapping regions of stitched image. 


\section{ACKNOWLEDGMENT}

This research work is financially supported by Savitribai Phule Pune University (SPPU), India through Assistance by SPPU for Project-based Innovative Research (ASPIRE) under Research Mentorship Program Scheme (18TEC001416) which is supervised by Internal Quality Assurance Cell (IQAC), SPPU, Pune, India.

\section{REFERENCES}

[1] Julio Zaragoza, Tat-Jun Chin, Quoc-Huy Tran, Michael S. Brown and David Suter, "As-Projective-As-Possible Image Stitching with Moving DLT", IEEE Transactions On Pattern Analysis And Machine Intelligence, Vol. 36, No. 7, pp. 1285 - 1298, July 2014. DOI: 10.1109/TPAMI.2013.247.

[2] Che Han Chang, Yoichi Sato and Yung Yu Chuang, "Shape preserving half projective warps for Image Stitching", IEEE Conference on Computer Vision and Pattern Recognition (CVPR), pp. 3254-3261, 2328 June 2014, Columbus, USA. DOI: 10.1109/CVPR.2014.422.

[3] Jing Li, Zhengming Wang, Shiming Lai, Yongping Zhai and Maojun Zhang, "Parallax-Tolerant Image Stitching Based on Robust Elastic Warping", IEEE Transactions on Multimedia, Vol. 20, No. 7, pp. 16721687, July 2018. DOI: 10.1109/TMM.2017.2777461.

[4] Chung-Ching Lin, Sharathchandra U. Pankanti, Karthikeyan Natesan Ramamurthy and Aleksandr Y. Aravkin, "Adaptive As-Natural-AsPossible Image Stitching", IEEE Conference on Computer Vision and Pattern Recognition (CVPR), pp. 1155-1163, 7-12 June 2015, Boston, USA. DOI: $10.1109 / C V P R .2015 .7298719$.

[5] Richard Szeliski, "Image Alignment and Stitching: A Tutorial", Foundations and Trends in Computer Graphics and Vision Journal, Vol. 2, No. 1, pp. 1-104, January 2006. DOI: 10.1561/0600000009.

[6] Lisa Brown, "A Survey of Image Registration Technique", ACM Computing Surveys (SCUR), Vol. 24, No. 4, pp. 325-376, December 1992. DOI: $10.1145 / 146370.146374$.

[7] Chia-Yen Chen, "Image Stitching - Comparisons and New Techniques", Computer Science Department, CITR, The University of Auckland, New Zealand, October 1998.

[8] David G. Lowe, "Distinctive Image Features from Scale-Invariant Keypoints", International Journal of Computer Vision (IJCV), Vol. 60, No. 2, pp. 91-110, November 2004. DOI: 10.1023/B:VISI.0000029664. 99615.94.

[9] Herbert Bay, Tinne Tuytelaars, and Luc Van Gool, "SURF: Speeded Up Robust Features", European Conference on Computer Vision (ECCV), pp. 410-417, 7-13 May 2016, Austria. DOI: 10.1007/11744023.

[10] Yan Ke and Rahul Sukthankar, "PCA-SIFT: A More Distinctive Representation for Local Image Descriptors", IEEE Conference on Computer Vision and Pattern Recognition (CVPR), pp. 506-513, 27 June - 2 July, 2004, Washington, USA. DOI: 10.1109/CVPR.2004. 1315206.

[11] Jian Wu, Zhiming Cui, Victor S. Sheng, Pengpeng Zhao, Dongliang Su and Shengrong Gong, "A Comparative Study of SIFT and its Variants", Measurement Science Review, Vol. 13, No. 3, pp. 122-131, June 2013. DOI: $10.2478 / \mathrm{msr}-2013-0021$.

[12] Omkar S. Vaidya, Dr. S. T. Gandhe and Pooja B. Sonawane, "Performance Analysis of KLT, Harris and SIFT Feature Detector for Image Stitching", International Journal of Electrical and Electronics Engineers (IJEEE), Vol. 8, No. 1, pp. 536-544, January-June 2016.
[13] M. Hassaballah, Aly Amin Abdelmgeid and Hammam A. Alshazly, "Image Features Detection, Description and Matching", Image Feature Detectors and Descriptors Foundations and Applications, Springer International Publishing, pp.11-45, Switzerland 2016. DOI: 10.1007/978-3-319-28854-3_2.

[14] Omkar S. Vaidya and Dr. Sanjay T. Gandhe, "The Study of Preprocessing and Postprocessing Techniques of Image Stitching", IEEE International Conference On Advances in Communication and Computing Technology (ICACCT), pp. 431-435, 8-9 February 2018, AVCOE, Sangamner, India. DOI: 10.1109/ICACCT.2018.8529642.

[15] Martin A. Fischler and Robert C. Bolles, "Random Sample Consensus: A Paradigm for Model Fitting with Apphcatlons to Image Analysis and Automated Cartography", Communications of the ACM, Vol. 24, No. 6, pp. 381-395, June 1981. DOI: 10.1145/358669.358692.

[16] Tat-Jun Chin, Jin Yu, and David Suter, "Accelerated Hypothesis Generation for Multi-Structure Robust Fitting", European Conference on Computer Vision (ECCV), pp. 533-546, 5-11 September, 2010, Greece. DOI: $10.1007 / 978-3-642-15555-0 \_39$.

[17] Richard Hartley and Andrew Zisserman, "Multiple View Geometry in Computer Vision", Second Edition, Cambridge University Press, 2003. DOI: $10.1017 / \mathrm{CBO} 9780511811685$.

[18] Junhong Gao, Seon Joo Kim and Michael S. Brown, "Constructing Image Panoramas using Dual-Homography Warping”, IEEE Conference on Computer Vision and Pattern Recognition (CVPR), pp. 49-56, 20-25 June 2011, Colorado Springs, USA. DOI: 10.1109/CVPR.2011.5995433.

[19] Wen-Yan Lin, Siying Liu, Yasuyuki Matsushita, Tian-Tsong Ng and Loong-Fah Cheong, "Smoothly varying affine stitching," IEEE Conference on Computer Vision and Pattern Recognition (CVPR), pp. 345-352, 20-25 June 2011, Colorado Springs, USA. DOI: 10.1109/CVPR.2011.5995314.

[20] Suiyi Ling, Gene Cheung and Patrick Le Callet, "No-Reference Quality Assessment for Stitched Panoramic Images using Convolutional Sparse Coding and Compound Feature Selection", IEEE International Conference on Multimedia and EXPO (ICME), pp. 1-6, 23-27 July, 2018, San Diego, USA. DOI: 10.1109/ICME.2018.8486545.

[21] Omkar S. Vaidya and Dr. Sanjay T. Gandhe, "Improvement in Image Alignment using Hybrid Warping Technique for Image Stitching", International Journal of Engineering Research and Technology (IJERT), Vol. 12, No. 3, pp. 350-356, March 2019.

[22] Anush Krishna Moorthy and Alan Conrad Bovik, "A Two-Step Framework for Constructing Blind Image Quality Indices", IEEE Signal Processing Letters, Vol. 17, No. 5, pp. 513-516, May 2010. DOI: 10.1109/LSP.2010.2043888.

[23] Anish Mittal, Anush Krishna Moorthy, and Alan Conrad Bovik, "NoReference Image Quality Assessment in the Spatial Domain", IEEE Transactions on Image Processing, Vol. 21, No. 12, pp. 4695-4708, December 2012. DOI: 10.1109/TIP.2012.2214050.

[24] Michele A. Saad, Alan C. Bovik and Christophe Charrier, "Blind Image Quality Assessment: A Natural Scene Statistics Approach in the DCT Domain", IEEE Transactions on Image Processing, Vol. 21, No. 8, pp. 3339-3352, August 2012. DOI: 10.1109/TIP.20122191563.

[25] Andrea Vedaldi and Brian Fulkerson, "VLFeat - An open and portable library of computer vision algorithms", International Conference on Multimedia, pp. 1469-1472, 25-29 October 2010, Italy. DOI: $10.1145 / 1873951.1874249$. 